Reprod. Nutr. Dévelop., 1981, 21 (5A), 695-704.

\title{
Etude expérimentale de l'action phagostimulante du saccharose et de la sinigrine et mise en évidence de phénomènes de régulation chez le criquet pèlerin Schistocerca gregaria (Orthoptère Acrididae)
}

par J. ASPIROT, Ginette LAUGÉ

Laboratoire d'Entomologie, Bâtiment 446

Université de Paris Sud, 91405 Orsay Cedex, France.

Summary. Experimental study of stimulatory effect of feeding sucrose and sinigrin in Schistocerca gregaria (Orthoptera : Acrididae) and regulation phenomena.

The stimulatory effect of feeding sucrose and sinigrin was studied with Vth instar larvae of Schistocera gregaria (Orthoptera). The food consisted of filter paper impregnated with the phagostimulant dissolved at different concentrations in water.

We studied the intake of filter paper and phagostimulant simultaneously and separately. The intake of sucrose or sinigrin-impregnated paper gradually increased up to a limit, depending on the concentration of the phagostimulant, then decreased. When the intakes of paper and of phagostimulant were studied separately, they evolved differently : phagostimulant intake remained constant at the highest concentrations as filter paper intake decreased. The stability of phagostimulant intake suggested regulatory processes whose nature has been discussed. The results with the two phagostimulants were comparable; however, they were obtained with lower amounts of sinigrin than of saccharose. There was no dissuasive or toxic effect even at every high concentrations.

\section{Introduction.}

L'acceptation d'un aliment par les insectes dépend des différentes substances phagostimulantes qu'il contient (Thorsteinson, 1960 ; Hsiao, 1969) et dont certaines ont une valeur alimentaire. Nous examinerons dans ce travail l'incidence, sur la prise de nourriture du Criquet pèlerin Schistocerca gregaria Forsk (Orthoptère Acrididae), de deux d'entre elles : le saccharose et la sinigrine, ainsi que les phénomènes qui interviennent lorsque, métabolisées, ces substances participent à l'alimentation du Criquet pèlerin.

Thorsteinson (1958), Dadd (1960), Sinoir (1969), Cook (1977) ont montré la valeur phagostimulante du saccharose chez les Acridiens. Mais le saccharose, dont les composantes participent au métabolisme de l'insecte, doit être considéré aussi comme une substance nutritive. Strebler (1978) signale que le saccharose ingéré par S. gregaria est en grande partie hydrolysé en glucose et fructose dans l'heure qui suit l'ingestion.

La sinigrine, thioglucoside caractéristique des Crucifères, sert de signal de reconnaissance pour les insectes inféodés à ces plantes. De nombreuses observations, faites 
sur le terrain et concernant le spectre alimentaire du Criquet pèlerin, signalent qu'en phase solitaire S. gregaria consomme parfaitement certaines Crucifères comme Farsetia ramosissima (Hochst), Farsetia aegyptiaca (Turra), Malcomia aegyptiaca (Spreng), Morettia canescens (Boiss), Schouwia purpurea (Forsk) (Sauvage, 1953 ; Chevassut ef Quetzel, 1956).

A partir de ces observations, Tira (1975), Le Berre et Tira (1977) ont supposé et effectivement mis en évidence l'action stimulante de la sinigrine sur la prise de nourriture du Criquet pèlerin, lorsque cette substance est ajoutée à un substrat inerte composé de papier filtre. Mais l'hydrolyse de la molécule de sinigrine fournit un radical glucose ; celui-ci peut donc éventuellement participer comme métabolite à l'alimentation du Criquet pèlerin. On est alors en droit de se demander si les phénomènes observés avec la sinigrine seront identiques ou non à ceux notés avec le saccharose. Ces phénomènes doivent se manifester plus particulièrement lors d'expériences de longue durée, permettant la métabolisation des deux molécules, saccharose ef sinigrine. Cette hypothèse a guidé le choix de la durée de nos expériences.

Enfin, nous nous sommes demandés si, au-delà des concentrations habituellement utilisées, l'effet phagostimulant du saccharose et de la sinigrine ne serait pas remplacé par un effet dissuadant, voire même toxique.

\section{Conditions expérimentales.}

Notre expérience a été conduite avec des femelles de Criquet pèlerin Schistocerca gregaria, du Ve stade et âgées de 2, 3 et 4 jours à l'intérieur du stade.

Les femelles sont mises à jeûner pendant $6 \mathrm{~h}$ avant d'être placées dans des cristallisoirs, par groupes de six, en présence d'un substrat inerte constitué de rondelles de papier filtre. Au moment de l'expérience, les cristallisoirs sonf disposés dans des enceintes sous un éclairement continu, à la fempérature de $33^{\circ} \mathrm{C} \pm 2{ }^{\circ} \mathrm{C}$ et avec une humidité relative de 50 p. 100 . Il n'y a aucun apport d'eau liquide durant toute l'expérience.

Les deux substances à étudier sont présentées sous forme de solution aqueuse de concentrations allant de 0,25 à 74 p. 100 en ce qui concerne le saccharose ef de 0,25 à 20 p. 100 en ce qui concerne la sinigrine. A l'aide d'une micropipette, un volume de $250 \mu l$ est déposé sur chaque rondelle de papier filtre, les rondelles témoins recevant pour leur part le même volume d'eau distillée. Les rondelles ayant reçu un volume connu de solution, il est possible de calculer leur charge en sucre ou sinigrine (tabl. 1). Ainsi préparées, les rondelles sont desséchées au dessiccateur sous vide à $50^{\circ} \mathrm{C}$ jusqu'à poids constant, afin d'éviter l'action phagostimulante de l'eau (Sinoir, 1969).

Une fois séchées, les rondelles sont groupées par concentration et pesées douze par douze, puis placées dans chaque récipient. Les quantités consommées sont évaluées par différence entre les poids secs de chaque rondelle avant et après l'expérience. Chaque lot d'insectes reste $18 \mathrm{~h}$ en présence de l'aliment; en effet, les criquets mis en présence de papier filtre imprégné de saccharose ont une activité alimentaire de plusieurs heures (10 h ou plus pour Locusta migratoria, Cook, 1976).

En ce qui concerne le saccharose, chacun des points de la gamme de concentra- 
tions a donné lieu à 12 répétitions (faibles concentrations) ou 5 répétitions (fortes concentrations). Pour des raisons pratiques, les 15 concentrations n'ont pu être expérimentées simultanément. Nous avons en fait réalisé une première série d'expériences avec des charges en sucre s'étendant de 0,48 à $76,88 \mathrm{mg}$ pour $100 \mathrm{mg}$ de papier filtre, puis une seconde série avec les très fortes teneurs en saccharose. La continuiłé des réponses a été vérifiée en reprenant dans les nouvelles séries expérimentales des concentrations en sucre déjà testées. Le très bon recoupement des résultats nous a autorisés à réunir l'ensemble des données. Les concentrations signalées dans les végétaux consommés par S. gregaria sont de 2 à 8 p. 100. Les concentrations les plus faibles choisies pour les solutions (préparées à température ambiante) sont donc inférieures à ces valeurs ; la plus forte concentration correspond à une solution proche de la safuration.

Chacun des points de la gamme de concentrations en sinigrine a donné lieu à 9 répétitions. Les concentrations expérimentées sont plus faibles qu'arec le saccharose : leur choix est discuté plus loin.

\section{TABLEAU 1}

Calcul de la charge en saccharose et en sinigrine pour $100 \mathrm{mg}$ de papier filtre, selon la concentration de la solution qui a servi à imprégner les rondelles

\begin{tabular}{cccccccccccc}
\hline & A SAC & 0,25 & 0,50 & 1 & 2,5 & 5 & 7,5 & 10 & & 15 & 20 \\
A & & & & & & & & & \\
SIN & 0,25 & 0,50 & 1 & 2,5 & 5 & 7,5 & 10 & 12,5 & 15 & 20 \\
B & 0,48 & 0,96 & 1,92 & 4,81 & 9,61 & 14,42 & 19,22 & 23,04 & 28,84 & 38,44 \\
A | SAC & 40 & 47 & 53 & 60 & 67 & 74 & & & & \\
B & 76,88 & 90,33 & 101,86 & 115,32 & 128,77 & 142,22 & & & & \\
\hline
\end{tabular}

A : Concentration de la solution aqueuse (en p. 100). SAC : saccharose ; SIN : sinigrine. B : Quantité de substance (en $\mathrm{mg}$ ) pour $100 \mathrm{mg}$ de papier filtre.

\section{Résultats.}

\section{Saccharose.}

Dans un premier temps, nous analyserons l'effet du saccharose sur la prise de nourriture. Les résultats représentés sur la figure 1 donnent les quantités consommées en fonction de la charge en sucre des rondelles.

Dès le seuil de 1 p. 100, le sucre augmente la prise de nourriture de façon hautement significative. La consommation de papier sucré croît progressivement en fonction des charges en sucre jusqu'à celle de 9,61 p. 100 ; elle est maximale et relativement stable entre les charges de 9,61 ef 38,4 p. 100 , puis diminue sensiblement pour les très fortes teneurs en sucre.

En première analyse, on serait tenté de considérer que le saccharose est bien phagostimulant, mais qu'il a un effet maximum pour une charge comprise entre 10 et 40 p. 100. Afin d'apporter un complément d'informations, nous avons estimé, d'après les résultats globaux, d'une part les consommations de papier ayant servi de support, d'autre part celles de saccharose. 


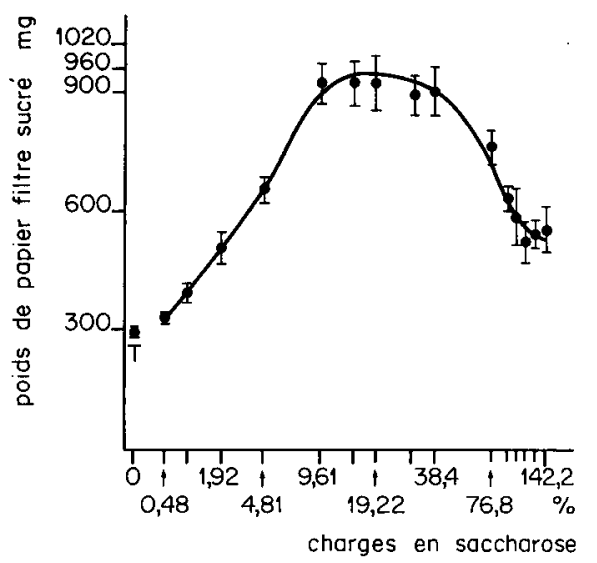

FIG. 1. - Quantifés de papier filtre sucré ingéré par des femelles de Ve stade de Schistocerca gregaria.

$T$ : femelles auxquelles on ne présente que du papier filtre (même signification pour les Figs. 2, 3,4). Remarques : l'échelle des abscisses est une échelle logarithmique ; les intervalles qui accompagnent chaque point sont les erreurs standard (E.S.).

Si l'on regarde ces quantités (fig. $2 a, b$ ), on note qu'il s'établit au fur et à mesure de l'augmentation des teneurs en sucre du substrat :
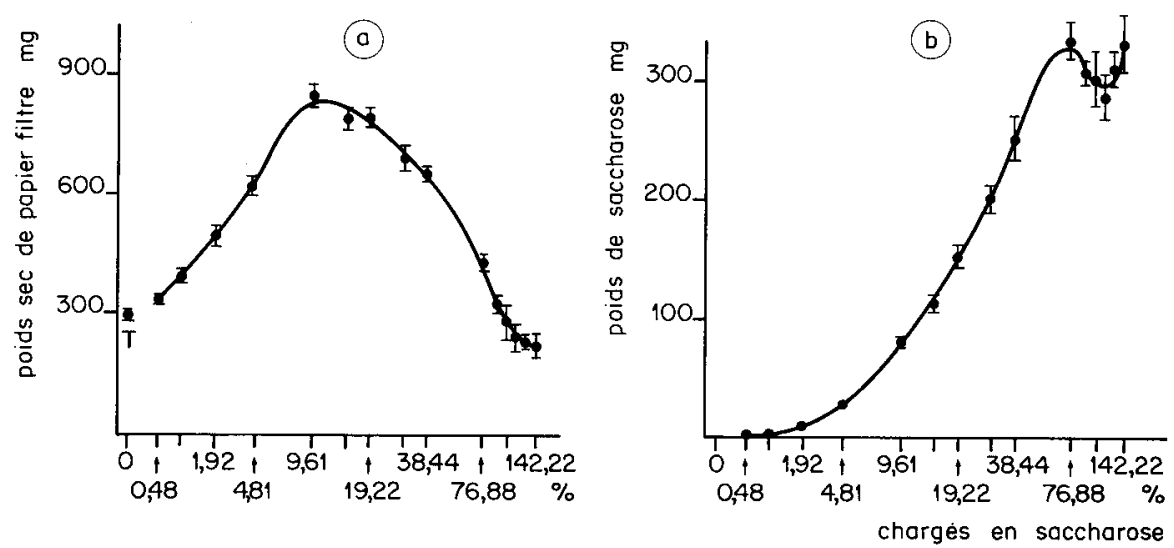

FIG. 2. - Quantités de papier filtre ef de saccharose consommés par des femelles de Ve stade de S. gregaria, auxquelles on présente des rondelles de papier sucré.

a : Quantités de papier filtre consommé; b : Quantités de saccharose consommé. Mêmes remarques que figure 1.

- une 1re phase au cours de laquelle l'augmentation des quantités de saccharose ingéré s'accompagne d'une prise de papier filtre de plus en plus importante. Cette phase s'étend de la charge 0,96 à la charge 9,61 ;

- une $2^{\mathrm{e}}$ phase où les quantités de papier filtre ingéré restent à peu près stationnaires, alors que la quantité de sucre ingéré continue d'augmenter. La quantité moyenne de papier filtre consommé est de $816,5 \mathrm{mg} \pm 26,95$;

- une $3^{\mathrm{e}}$ phase où l'on constate que les quantifés de sucre ingéré continuent de progresser tandis que celles du papier filtre ingéré diminuent ; par suite, la consommation globale de papier imbibé de saccharose apparaît stable (fig. 1). La comparaison 
entre la consommation moyenne pendant la deuxième phase et celle qui marque le début de la troisième phase donne une différence significative au seuil de 5 p. 100 $(t=2,34)$;

- une $4^{\mathrm{e}}$ phase correspond aux très fortes teneurs en sucre, supérieures à 38,44 p. 100 . Ici, la consommation de papier filtre diminue progressivement, alors que les quantités de sucre ingéré restent stables. Il n'y a pas de différence significative entre les quantités de sucre ingéré aux différentes concentrations : on peut donc assimiler cette phase à un plateau. La consommation moyenne calculée $(318,24 \mathrm{mg} \pm 9,58)$ donne une différence hautement significative au seuil de 1 p. $100(\dagger=3,30)$ par rapport à la consommation en sucre pour la teneur de 38,44 p. 100.

Les résultats sont donc totalement différents selon que l'on s'intéresse à la quantité de substance chimique consommée (fig. $2 b$ ) ou à la quantité de substrat que cette substance a incité à consommer (fig. $2 a$ ).

\section{Sinigrine.}

La figure 3 donne les quantités consommées par le Criquet pèlerin en fonction de la charge du substrat en sinigrine. A la différence de l'expérience précédente où les quantités de saccharose déposées sur les rondelles allaient de 0,48 à $142,2 \mathrm{mg}$ pour $100 \mathrm{mg}$ de papier, la gamme des teneurs en sinigrine est nettement plus restreinte et n'excède pas $38,44 \mathrm{mg}$. Il y a d̀ cela deux raisons :

- d'une part, les teneurs choisies entraînent des variations effectives de la prise de nourriture,

- d'autre part, le coût élevé de la substance n'a pas permis d'expérimenter l'action de concentrations plus élevées.

Avec cette gamme, nous observons une augmentation de la prise de nourriture significative au seuil de 1 p. 100 dès la première teneur en sinigrine proposée aux criquets. La consommation croît ensuite rapidement jusqu'à la teneur 1,92 p. 100, puis devient stable entre 1,92 et 4,81 avant de diminuer fortement (fig. 3). A partir de la teneur 19,22 p. 100, la consommation de papier imprégné de sinigrine n'est plus quantitativement différente de la consommation sur papier filtre seul (témoin).

FIG. 3. - Quantités de papier filtre imprégné de sinigrine, ingéré par des femelles de $V^{e}$ stade de S. gregaria Mêmes remarques que figure 1 .

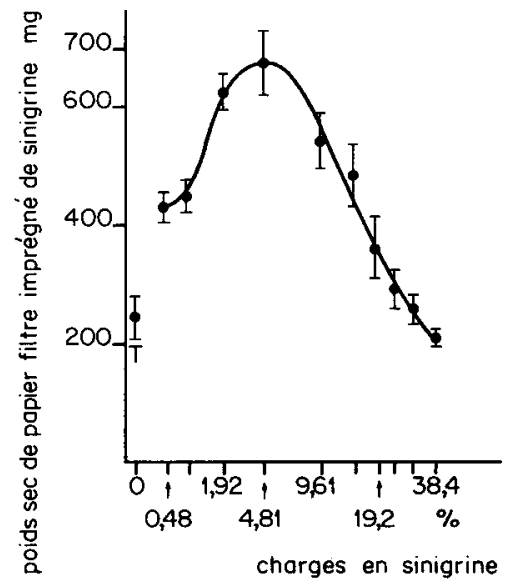


Comme précédemment, nous avons estimé la consommation de papier ayant servi de support d'une part et celle de sinigrine d'autre part. La séparation des deux composantes, papier filtre et sinigrine, nous permet de mettre en évidence un phénomène identique à celui déjà observé avec le saccharose. En fonction des concentrations, nous retrouvons 4 phases (fig. $4 a, b$ ).

- Aux faibles teneurs en sinigrine, l'augmentation des quantités de sinigrine ingérée s'accompagne d'une prise de papier filtre de plus en plus importante. L'effet phagostimulant est conditionné par la charge en sinigrine des rondelles. Cette étroite relation qui existe entre les quantités de sinigrine ingérée et la prise de nourriture ne se modifie pas jusqu'à la teneur 1,92 p. 100.

- Une $2^{\text {e }}$ phase limitée, qui s'étale de la teneur 1,92 à 4,81 p. 100 où les quantités de papier filtre ingéré sont stables alors que la quantité de sinigrine ingérée continue de progresser. La moyenne de ce plateau est de $640,15 \mathrm{mg}$.
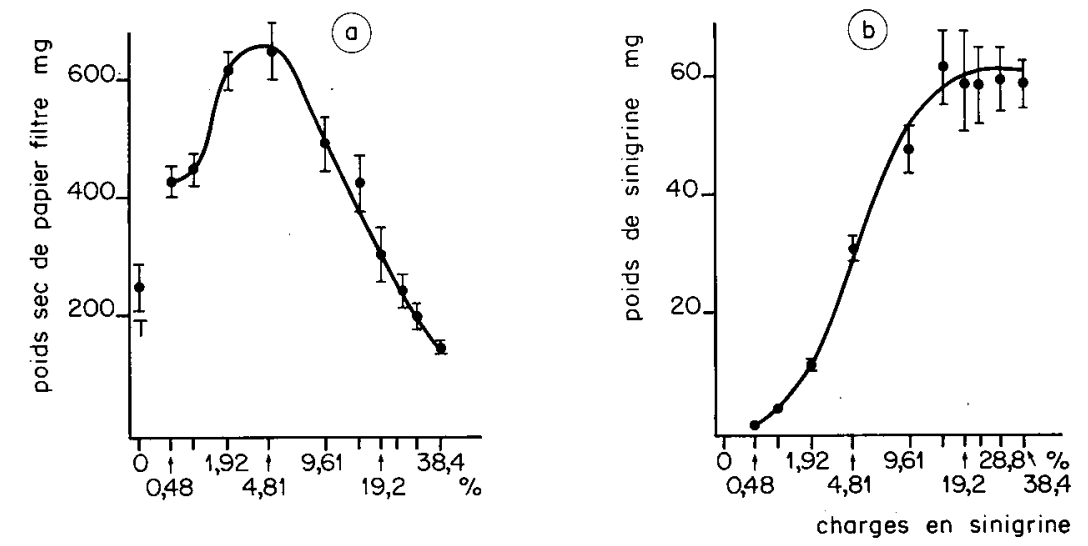

FIG. 4. - Quantités de papier filtre et de sinigrine consommés par des femelles de Ve siade de S. gregaria, auxquelles on présente des rondelles de papier imprégné de sinigrine.

a : Quantités de papier filtre consommé, b : Quantités de sinigrine consommée. Mêmes remarques que figure 1.

- Une $3^{e}$ phase apparaît, lorsque la charge en sinigrine dépasse la teneur de 4,81 p. 100. En effet, alors que la consommation de papier diminue fortement (fig. 4a), les quantités de sinigrine ingérée continuent de progresser mais de façon moindre (fig. $4 b$ ). Par la suite la consommation globale diminue (fig. 3 ).

La comparaison entre la consommation moyenne pendant la $2^{\mathrm{e}}$ phase et celle qui marque le début de la $3^{\mathrm{e}}$ phase donne une différence hautement significative au seuil de 1 p. 100.

- Une $4^{\mathrm{e}}$ phase correspond aux charges en sinigrine les plus élevées. Les quantités de sinigrine ingérée restent constantes, bien que les rondelles supportent des charges de plus en plus fortes.

Comme pour le saccharose, les résultats sont donc totalement différents selon que l'on s'intéresse à la quantité de substance consommée (fig. $4 b$ ) ou à la quantité de substrat que cette substance a incité à consommer (fig. 4a).

Ces expériences montrent donc que $S$. gregaria se comporte vis-à-vis des deux 
substances étudiées d'une manière semblable : la prise de papier filtre est une fonction croissante de la charge des rondelles en sinigrine ou en saccharose, jusqu'au seuil au-delà duquel l'augmentation de la teneur en ces différentes substances cesse de faire croître la prise de nourriture et même tend à la diminuer. Le calcul des quantités de substances ingérées montre qu'aux plus fortes charges utilisées, la substance phagostimulante a été absorbée en quantité limitée et constante, faisant corrélativement diminuer la consommation de papier filtre imprégné de la substance à éprouver. Notons que les phénomènes observés avec la sinigrine ont lieu pour des teneurs très inférieures à celles relevées avec le saccharose.

\section{Discussion.}

Les expériences que nous venons de décrire monirent que $S$. gregaria module sa consommation en fonction du caractère plus ou moins phagostimulant de l'aliment.

Nous constatons que la consommation globale de papier sucré ou de papier imprégné de sinigrine augmente progressivement, se maintient stable, puis diminue pour les fortes teneurs. Divers auteurs ont montré qu'il existe une relation entre la concentration de la substance et la quantité d'aliment ingéré. Cette relation sert à mesurer la phagostimulation. Rappelons que nos expériences ont lieu sans apport supplémentaire d'eau. La présence d'eau aurait très certainement entraîné une modification de la prise de nourriture (Sinoir, 1969), mais il est probable que cette modification serait intervenue de manière comparable dans les deux séries d'expériences.

Les stimuli chimiques perçus par les récepteurs sensoriels préoraux, non seulement guident les insectes dans la reconnaissance de la plante hôte, mais contrôlent la quantité de nourriture prise. Ma (1972) et Blom (1978) constatent, sur des chenilles de Lépidoptères par exemple, l'existence d'une relation entre la fréquence des potentiels d'action enregistrés au niveau des récepteurs sensoriels sensibles au sucre ef la concentration molaire des solutions utilisées pour stimuler ces récepteurs. Ces auteurs ont trouvé également une relation entre la fréquence des potentiels d'action et la réponse comportementale de l'insecte ; en d'autres termes, les quantités ingérées sont en rapport avec l'intensité de la stimulation des récepteurs sensoriels.

Dans nos expériences, la prise de nourriture augmente en fonction de la concentration jusqu'à la valeur 9,61 p. 100 pour le saccharose et 1,92 p. 100 pour la sinigrine. Mais aux teneurs plus fortes, les quantités ingérées ne croissent plus avec le gradient des charges. Ce résultat pose le problème de l'interprétation des courbes dans la partie correspondant aux fortes concentrations.

Nous examinerons successivement le cas du saccharose, puis celui de la sinigrine.

Saccharose. - Si l'on dissocie les quantités globales ingérées en leurs composantes : papier filtre d'une part et substance phagostimulante (le sucre) d'autre part, on observe que la quantité de substance ingérée augmente, puis demeure constante au-delà de la teneur de 76,88 p. 100. Aux faibles et aux moyennes teneurs, la concentration de la substance ef la quantité de substance ingérée augmentent simultanément; phénomène qui est la traduction de l'effet phagostimulant du saccharose. Aux fortes teneurs, cette relation n'existe plus ef la stabilité de la consommation suggère l'existence d'un processus de régulation. 
Une première hypothèse explicative est celle d'une régulation de la consommation par saturation du système sensoriel : le nombre de sensilles concernées pourrait être de plus en plus important, jusqu'à ce qu'une concentration suffisamment forte stimule l'ensemble des récepteurs sensoriels, déterminant ainsi un maximum dans l'excitation qui entraînerait un blocage de la consommation.

D'autres hypothèses peuvent être avancées :

- Selon Bernays, Blaney et Chapman (1972), les afférences sensorielles extéroceptives interviendraient au niveau des phénomènes de régulation durant la consommation, par ouverture et fermeture des voies d'accès aux sensilles : avant le repas, les sensilles sont ouvertes, donc fonctionnelles ; après le repas, elles sont obturées par un mucus, donc non fonctionnelles. Ce processus pourrait avoir une base hormonale. - Selon les travaux de Dethier et al. (1956) chez Phormia regina et de Treherne (1957) chez Periplaneta americana, les quantités de nourriture ingérée peuvent être modulées par des mécanismes internes assurant la régulation du transit alimentaire : vitesse de vidange du jabct qui se fait de plus en plus lentement lorsque la concentration en glucose est de plus en plus forte.

- Enfin, la pression osmotique de l'hémolymphe est un facteur de régulation possible. Bernays et Chapman (1974) ont montré qu'en augmentant la pression osmotique par injection de tréhalose chez Locusta migratoria, la prise de nourriture est réduite. Dans son étude sur la phagostimulation chez Locusta migratoria, Cook (1977) considère la pression osmotique de l'hémolymphe comme un facteur important dans la régulation des quantités globales ingérées. Enfin, chez Phormia regina, le passage rapide des sucres au travers de la paroi intestinale modifie la pression osmotique de l'hémolymphe et se répercute sur la vitesse du transit alimentaire (Gelperin, 1966).

Dans les conditions normales, la vitesse de l'hydrolyse des glucides est rapide : 82,1 p. 100 du saccharose ingéré par $S$. gregaria est hydrolysé en glucose et fructose dans l'heure qui suit l'ingestion (Strebler, 1978). Par conséquent, dans nos expériences, le saccharose consommé pendant $18 \mathrm{~h}$ a eu le temps d'être hydrolysé et assimilé, modifiant ainsi, selon les hypothèses des auteurs, les équilibres internes qui assurent la régulation de la prise de nourriture.

On peut penser que tous ces phénomènes de régulation sensorielle, ou interne, interfèrent les uns sur les auirres. Dès lors, il serait vain d'interpréter nos résultałs concernant la prise de nourriture comme la seule conséquence du pouvoir phagostimulant du sucre, alors que la durée de l'expérience est de $18 \mathrm{~h}$.

Sinigrine. - Les expériences avec la sinigrine ont donné des résultats similaires à ceux obtenus avec le saccharose. Mais la stabilisation des quantités de substance ingérée s'observe à des teneurs nettement plus faibles, à partir de 14,42 p. 100. On peut, comme précédemment, envisager une régulation de type sensoriel pour laquelle les sensilles concernées seraient soit inférieures en nombre et différentes de celles sensibles au saccharose, soit (dans le cas où les mêmes sensilles seraient stimulées) manifesteraient un potentiel d'action différent.

Dans la mesure où $S$. gregaria possède une $\beta$ glucosidase et étant donné que la sinigrine peut être hydrolysée en libérant du glucose et une molécule d'isothiocyanate (Ettlinger et Kjaer, 1968 ; Wittacker et Feeny, 1971 ; Finch, 1978), nous pouvons émettre l'hypothèse que la sinigrine est partiellement absorbée au niveau de la muqueuse 
intestinale. Le glucose assimilé peut, comme celui provenant du saccharose, réguler la prise de nourriture en modifiant les équilibres internes. On doit néanmoins constater que les phénomènes de régulation interviennent ici plus tôt que dans l'expérience précédente. II est donc vraisemblable que la molécule d'isothiocyanate, libérée lors de l'hydrolyse de la sinigrine, joue un rôle par ses radicaux soufrés et cyanurés.

Enfin, l'allure des courbes obtenues avec la sinigrine et le saccharose étant la même, il paraît difficile de parler d'effet toxique. Toutefois, le problème reste ouvert.

\section{Conclusion.}

Suite à nos expériences, il apparaît délicat de discuter de la valeur phagostimulante des diverses concentrations de saccharose ou de sinigrine sans tenir compte des phénomènes de régulation qui interviennent pendant la durée de l'expérience. Ainsi, la stimulation de plus en plus élevée due à l'augmentation de la concentration entraine une consommation progressivement croissante ; très tôt, des processus de régulation viennent s'ajouter, faisant varier la prise de nourriture et ne permettant plus d'établir une relation simple. Devant ces difficultés d'interprétation, d'autres critères devraient être choisis de façon à pouvoir mieux comprendre l'effet phagostimulant des substances primaires assimilables (saccharose) et des substances secondaires peut-être assimilables (sinigrine). Nos expériences montrent que la quantité de substrat consommé obéit, dans les deux cas, aux mêmes règles.

A partir d'un certain seuil, les quantités ingérées restant constantes quelle que soit la concentration de la substance mise en expérience, nous pouvons conclure qu'il n'y a vraisemblablement pas de phénomène de répulsion, ni de toxicité.

$$
\begin{aligned}
& \text { Reçu en janvier } 1981 . \\
& \text { Accepté en avril } 1981 .
\end{aligned}
$$

\section{Références}

BERNAYS E. A., BLANEY W. A., CHAPMAN R. F., 1972. Changes in chemoreceptor sensilla on the maxillary palps of Locusta migratoria in relation to feeding. J. exp. Biol., 57, 745-753.

BERNAYS E. A., CHAPMAN R. F., 1974. Changes in haemolymph osmotic pressure in Locusta migratorio larvae in relation to feeding. J. Ent. (A), 48, 149-155.

BLOM F., 1978. Sensory input behavioural output relationships in the feeding activity of some Lepidopterous larvae, 258-263. Proc. 4 th int. Symp. Insect and Host Plant, R. F. CHAPMAN, E. A. BERNAYS, Ned. Ent. Ver. Amsterdam.

CHEVASSUT G., QUETZEL P., 1956. Récoltes botaniques au Tibesti. Off. nat. anti-acridien, Bull. $n^{0} 7$. COOK A. G., 1976. A critical review of the methodology and interpretation of experiments designed to assay the phagostimulatory activity of chemicals to phytophagous insects. Symp. biol. hung., 16, 47-54.

COOK A. G., 1977. Nutrient chemicals as phagostimulants for Locusta migratoria (L.). Ecol. Entomol., 2, 113-121.

DADD R. H., 1960. Studies on the nutrition of locusts. III. Carbohydrate requirements and utilization. J. Insect Physiol., 5, 301-316.

DETHIER V. G., EVANS D. R., RHOADES M. V., 1956. Some factors controlling the ingestion of carbohydrates by the bowfly. Biol. Bull. mar. Biol. Lab. Woods Hole, 111, 204-222.

ETTLINGER M., KJAER A., 1968. Sulfur compounds in plants. Rec. Adv. Phyfochem. T. L. MARBRY, R. E. ALSTON et V. C. RUNECKLES, Appleton-Century Crofts, New York, 1, 59-144.

FINCH S., 1978. Volatile plant chemicals and their effect on host plant finding by the cabbage root fly (Delia brassicae), 350-359. Proc. 4th int. Symp. Insect and Host Plant, R. F. CHAPMAN, E. A. BERNAYS, Ned. Ent. Ver. Amsterdam. 
GELPERIN A., 1966. Control of crop empting in the bowfly. J. Insect Physiol., 12, 331-345.

HSIAO T. H., 1969. Chemical basis of host selection and plant resistance in oligophagous insects. Ent. exp. appl., 12, 777-778.

LE BERRE J. R., TIRA R., 1977. Stimulation, par la sinigrine, de la prise de nourriture du criquef pèlerin Schistocerca gregaria Forsk. (Orthoptera Acrididae). C. R. Acad. Sci. Paris, Sér. D, 284, $573-$ 576.

MA W. C., 1972. Dynamics of feeding response in Pieris brassicae (Linn.) as a function of chemosensory input : a behovioural, ultrastructural and electrophysiological study. Th. Wageningen, 72-11, $162 \mathrm{PP}$.

SAUVAGE Ch., 1953. Les récoltes botaniques des missions de l'Office national anti-acridien au Sahara occidental. Off. nat. anti-acridien, Bull. $n^{\circ} 4$.

SINOIR Y., 1969. Contribution à l'étude de la prise de nourriture chez la larve du criquet migrateur Locusta migratoria migratorioides (R. et F.) Orthoptère Acrididae. Th. Etat, Univ. Paris-Sud, Orsay.

STREBLER G., 1978. L'activité osidasique des enzymes digestifs de Schistacerca gregaria Forsk. Données quantitatives nouvelles. Ann. Zool. Ecol. anim., 10, 85-96.

THORSTEINSON A. J., 1958. Acceptability of plants for phytophagous insects. Proc. 10th inf. Congr. Entomol., Montreal, 2, 599-602.

THORSTEINSON A. J., 1960. Host selection in phytophagous insects. Ann. Rev. Ent., 5, 193-218.

TIRA R., 1975. Elevage du criquet pèlerin Schistocerca gregaria Forsk. sur milieu nutritif artificiel. Son intérêt en entomologie appliquée. Th. Doct. Ing., Univ. Paris-Sud, Orsay.

TREHERNE J. E., 1957. Glucose absorption in the cockroach. J. exp. Biol., 35, 862-870.

WITTACKER R. H., FEENY P. P., 1971. Allelochemics : chemical interaction between species. Science, 171, 757-770. 